\title{
Long Term Function Following the Central Cord Syndrome
}

\author{
M. J. Bridle, MA, OTR, K. B. Lynch, PhD, C. M. Quesenberry, OTR \\ The Virginia Regional Spinal Cord Injury Center, Department of Orthopaedics \\ and Rehabilitation, Box 426, University of Virginia Medical Center, Charlottes- \\ ville, Virginia, USA.
}

\section{Summary}

Follow-up visits were made to persons with central spinal cord injury in order to assess long term functional outcomes. An occupational therapist conducted in-home evaluations of 18 randomly selected subjects at an average time of 4 years 8 months since injury. Evaluation instruments included: the ASIA Motor Index; the febsen Hand Function Test; the Klein-Bell Activities of Daily Living Scale; the Feeling Tone Checklist; the Multidimensional Pain Inventory (MPI) and the Occupational Performance History Interview (OPHI)-Research Version. Spontaneous improvement in bowel and bladder function and ambulation was observed in all subjects. Despite poor hand function, subjects compensated through use of adaptive equipment to perform activities of daily living well. Most subjects reported experiencing pain and fatigue, many of them on a daily basis. A statistically significant difference was found between males and females (with females performing better) on the MPI's scales measuring interference of pain with household chores $(\mathrm{p}<0.005)$ and general activity level $(\mathrm{p}<0.05)$. A statistically significant difference $(\mathrm{p}<0.005)$ was also noted in adaptation to daily life before and after injury, as measured by the OPHI. The study results suggest that: (1) the problems of pain, fatigue and environmental barriers may not be recognised until patients have been living at home for some time; (2) central cord injured persons may benefit from training in pain management and energy conservation techniques subsequent to discharge from their inpatient rehabilitation programme; (3) visits should be made to the homes and work sites of central cord injured persons subsequent to their discharge to assess and alleviate environmental barriers.

Key words: Central spinal cord injury; Function; Pain; Fatigue.

Approximately $16 \%$ of patients with cervical spine injuries present with central cord syndrome (Merriam et al., 1986) while about $70 \%$ of all incomplete cer- 
vical spine injuries are central cord lesions (Bosch et al., 1971). This syndrome was first described by Schneider et al. in 1954. Major characteristics of the syndrome include: (1) more severe motor involvement in the upper extremities than the lower; (2) urinary retention; and (3) varying degrees of sensory impairment below the lesion. Where there is return of function following the injury it occurs in predictable sequence with the lower extremities recovering first, followed by recovery of bladder function, strength in the upper extremities, and last, finger movement. This pattern of recovery was subsequently explained by Hardy and Rossier (1975) who described a pattern of receding medullary oedema.

The prognosis for central cord injury is considered to be good (Merriam et $a l .$, 1986). However, there is a dearth of information about the long term effects of central cord injury on physical functioning and adaptation to daily life after the injury.

The purpose of the study was to evaluate the long term functional outcomes of persons with central spinal cord injury. Specifically, in view of the characteristics of the central cord syndrome, the authors were interested in assessing subjects' fine finger movement and performance of activities of daily living. Because pain is a common complication of spinal cord injury (Burke, 1973; Donovan et al., 1982; Nepomuceno et al., 1979) and persons with incomplete lesions may be particularly susceptible to it (Grundy and Russell, 1986), perception of pain was also examined. In addition, as fatigue is a potential side-effect of any long term disability, subjects' perceptions of fatigue were assessed. Finally, comparisons were made of subjects' adaptation in such areas as daily living routines, productivity, and leisure before and after the occurrence of the spinal cord injury.

\section{Patients and methods}

Twenty subjects were randomly selected from a pool of 43 patients who had been treated at a regional hospital. Eighteen of the 20 agreed to participate in the study. The mean time since injury was 4 years and 8 months, with the range being 2 years 8 months to 9 years 8 months.

Each subject was visited at home by an occupational therapist who administered the following assessments:

1. The ASIA Motor Index (ASIA, 1982) is a classification system based on ten key muscles associated with the significant neurological levels of the spinal cord. Using a manual muscle test the muscles are graded on a scale of 0 to 5, with 0 indicating total paralysis and 5 indicating normal strength. Total scores can range from 0 to 100 .

2. The Jebsen Hand Function Test (Jebsen et al., 1969) (JHFT) requires the person to perform 7 tasks designed to represent various hand activities, and the time taken to complete each task is recorded. The test was standardised on 390 persons with normal hand functioning. Thus, subjects' task completion times may be converted to standard scores to faciliate interpretation of results. The higher the standard score, the poorer the hand function; a standard score of 0 indicates normal hand functioning. 
Table I Multidimensional pain inventory scales

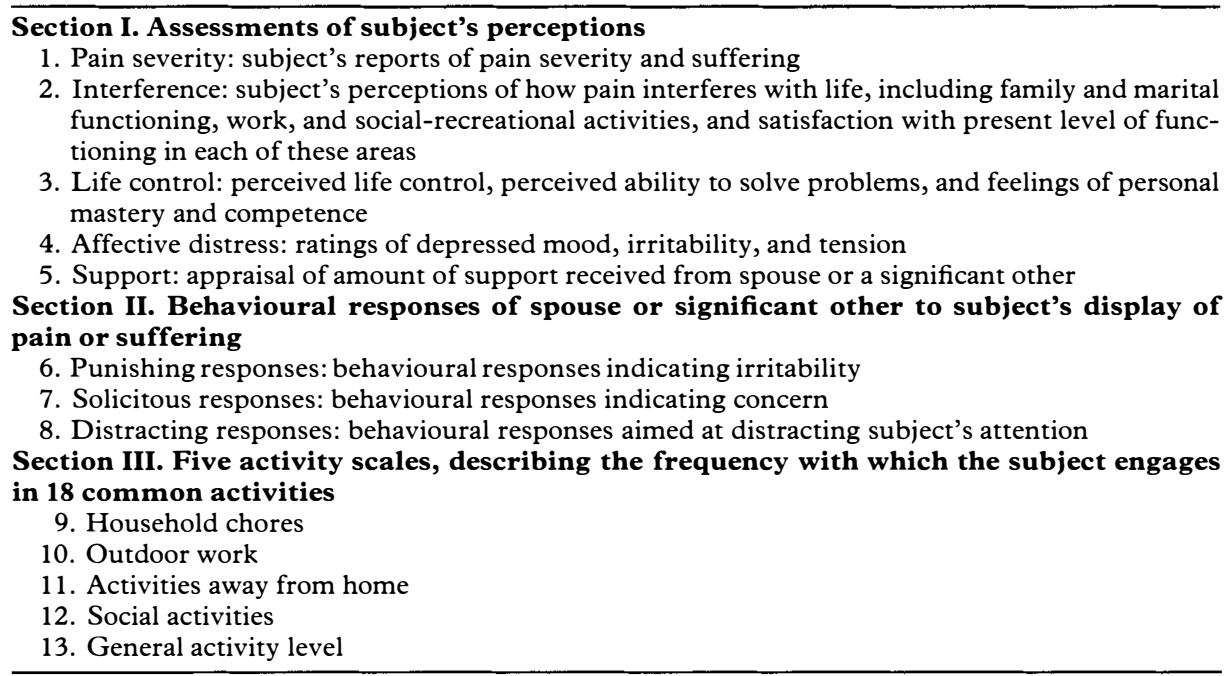

3. The Klein-Bell Activities of Daily Living (ADL) Scale (Klein and Bell, 1982) assesses the person's independence in 6 areas of self-care: dressing, elimination, mobility, bathing/hygiene, eating and emergency communication. Scores can range from 0 to $100 \%$ on each of the 6 subscales, and an overall mean score is calculated from these subscale scores. There is no penalty in scoring for using adaptive equipment. The inter-rater reliability of the Klein-Bell ADL Scale is $92 \%$; preliminary data indicate that the scale is a valid measure.

4. The Feeling Tone Checklist (Pearson and Byars, 1956) (FTC) is a subjective measure of fatigue. Respondents rate whether they feel 'Better than', 'Same as' or 'Worse than', 10 statements reflecting various states of fatigue or energy. Scores can range from 10 to 30 with 10 indicating minimum fatigue and 30 indicating maximum fatigue. Discriminant validity and internal consistency of the scale have been demonstrated, and the coefficient of reproducibility is 0.93 .

5. The Multidimensional Pain Inventory (Turk and Rudy, 1987) (MPI) measures subjects' perceptions of pain severity and the impact of pain on their lives. The MPI comprises 13 scales composed of varying numbers of items. Internal consistency and test-retest reliability were demonstrated for the scales. Brief descriptions of the scales are presented in Table I.

Each subject's pattern of response on the MPI may be used to classify the person into 1 of 4 profile types which were empirically derived from studies of chronic pain patients. These classifications, and characteristics associated with them, are as follows: dysfunctional (patients who report severe pain which interferes a great deal in many aspects of their lives); interpersonally distressed (patients who report an unusually high level of interpersonal distress); adaptive coper (patients who report high levels of social support, relatively low levels of pain and interference with their lives, and greater amounts of activity and per- 
cieved life control); anomalous (respondents who fit none of these profiles (Turk and Rudy, 1988).

The MPI was externally validated against other self-report and behavioural measures of pain. Concurrent and construct validity were established. The accuracy of the MPI scales in categorising patients into profile types was assessed by computing Cohen's kappa reliability coefficient (Cohen, 1960); results indicated that the MPI classification procedure made $97.5 \%$ fewer errors than would be expected by random assignment of patients into profile types.

6. The Occupational Performance History Interview-Research Form (Kielhofner and Henry, 1988) (OPHI) was designed to gather an individual's work, play and self-care performance history. The interview covers 5 areas as follows: environmental influences; life roles; organisation of daily routines; perceptions of control and ability; and interests, values and goals. Taken together these 5 areas provide an holistic picture of the individual's process of adapting in everyday occupational performance.

\section{Description of the sample}

Of the 18 subjects, 3 were female and 15 male. The mean age was 52 years (range 26-70). Injuries were sustained from $\mathrm{C} 1$ to $\mathrm{T} 1$ with the majority occurring at C5. On admission to hospital 5 of the subjects were classified as having a complete injury and the remainder incomplete. The diagnosis of complete lesion was changed before discharge as these subjects evidenced return of function in the subacute stage. Thus all 18 subjects were classified as having incomplete injuries at discharge.

Treatment included stabilisation of the spine followed by standard medical care and rehabilitation. The spines of 7 subjects were stabilised using a halo vest; 3 of these had surgery as well. The spines of the remaining 11 subjects were stabilised with a cervical collar, usually a Philadelphia collar. Medical care included treatment of any associated injuries such as abrasions, fractures and internal injuries as well as treatment for any complications such as infections. Rehabilitation included occupational and physical therapy with a primary focus on activities of daily living, ambulation and general strengthening. Two of the subjects were provided with orthoses to enhance hand function but discarded these immediately on discharge. None of the subjects had hand surgery.

Prior to injury 13 subjects were employed full-time, 3 part-time, 1 retired and 1 was a student. At follow-up 5 were employed full-time (4 of whom had returned to the same job), 3 were part-time, 3 retired and 7 unemployed. Thus, there was an overall decrease in employment following injury.

In all but 1 case, ambulation improved between discharge from the hospital and follow-up. At discharge, 8 subjects ambulated independently, 2 used a walker, 7 used a cane, and 1 used crutches. At follow-up 10 were walking independently, 7 used a cane and 1 used a wheelchair as a result of loss of lower limb function subsequent to discharge.

Bowel and bladder function also improved over time. At discharge 8 subjects were continent in bowel and bladder, while the remaining 10 were incontinent for both functions. At follow-up 13 were continent in bowel and bladder, 2 had 
Table II Klein-Bell ADL scale subscale scores

\begin{tabular}{lr}
\hline \multicolumn{1}{c}{ Activity } & Mean \\
\hline Dressing & $90 \%$ \\
Elimination & $92 \%$ \\
Mobility & $96 \%$ \\
Bathing/hygiene & $94 \%$ \\
Eating & $98 \%$ \\
Emergency communication & $100 \%$ \\
\hline
\end{tabular}

bowel incontinence with bladder continence and 3 remained incontinent for both functions. These improved outcomes in ambulation and elimination are characteristic of the syndrome (Schneider et al., 1954).

\section{Results}

The mean ASIA Motor Index score was 81 (range 56-96), out of a maximum possible score of 100 , suggesting reasonably good overall motor function. However, the mean standard score for the JHFT was 9.5 (range 4.02-18.09) indicating poor to very poor hand function. When the upper extremity (UE) ASIA Motor Index scores were separated from the lower extremity (LE) scores the mean for the LE was higher ( $\mathrm{UE}=38$; $\mathrm{LE}=43$ ) but this difference was not statistically significant. Correlation analysis of the mean UE ASIA score and the mean JHFT score resulted in a statistically significant negative correlation $r=-0.60, p<0.01$; whereas the same analysis of the JHFT and the mean LE ASIA score was not statistically significant, $r=-0 \cdot 34$. (Recall that a high score on the JHFT indicates poor function while a high score on the ASIA indicates good function).

Despite the poor hand function indicated by the JHFT, the mean total score on the Klein-Bell ADL scale was 93\% (range 66-100\%). This high level of function was reflected across the 6 subscales (Table II). Closer examination of the data revealed that 11 of the subjects $(61 \%)$ compensated for lost hand function by using adaptive equipment such as button hooks and dressing sticks (recall that the Klein-Bell does not penalise for equipment use).

Fifteen of the 18 subjects $(83 \%)$ reported feeling fatigue, with 5 persons $(27 \%)$ complaining of fatigue every day, all day. Approximately half the subjects attributed their fatigue to the spinal cord injury. The mean score on the FTC was $19 \cdot 5$, about the mid-point of the range of possible scores.

Thirteen subjects $(72 \%)$ complained of pain, with 12 persons $(66.6 \%)$ stating that they experience pain 24 hours a day, every day. Despite this only 3 respondents reported taking medication for pain. T-test analysis of data from the MPI revealed a statistically significant difference between the female and male subjects with respect to pain interfering with household chores $(p<0.005)$ and general activity level $(p<0.05)$. Despite pain the females continued to participate in these activities. It was thought this result might be explained in part if the women subjects suffered less fatigue than their male counterparts, but the data did not support this hypothesis.

Using the MPI classification system, the 13 subjects who completed the MPI 
Table III Comparison of subjects' pain profile types with MPI development sample

\begin{tabular}{lllll}
\hline & \multicolumn{2}{l}{$\begin{array}{l}\text { Study sample }(n=13)^{\star} \\
\text { Persons with central cord injuries }\end{array}$} & \multicolumn{2}{l}{$\begin{array}{l}\text { MPI sample }(n=22) \\
\text { Persons with chronic pain }\end{array}$} \\
\cline { 2 - 3 } Profile type & $\mathrm{n}$ & $\%$ & $\mathrm{n}$ & $\%$ \\
\hline Dysfunctional & 2 & 15.4 & 93 & 41.9 \\
Interpersonally distressed & 2 & 15.4 & 65 & 29.3 \\
Adaptive coper & 7 & 53.8 & 59 & 26.6 \\
Anomalous & 2 & 15.4 & 5 & 2.2 \\
\hline
\end{tabular}

* Four of the 18 subjects reported having no pain, and 1 did not complete the MPI.

Table IV Occupational performance history rating scales. Differences in adaptation before and after injury. Statistically significant t-test results

\begin{tabular}{ll}
\hline \multicolumn{1}{c}{ Scale } & Probability \\
\hline Organisation of daily living routines & $p<0.01$ \\
Life roles & $p<0.05$ \\
Interests, values, and goals & $p<0.001$ \\
Perception of ability and responsibility & $p<0.05$ \\
Environmental influences & $p<0.01$ \\
\hline
\end{tabular}

$N=18$ for all t-tests.

were assigned to pain profile types as follows: 2 dysfunctional, 2 interpersonally distressed, 7 adaptive coper, and 2 anomalous. This distribution of profile types differed from that found in the original MPI validation studies, as can be seen in Table III. Notably, a larger percentage of subjects from this study (persons with central cord injuries) were classified as adaptive copers, as compared with the chronic pain patients from whose responses the profile types were empirically derived.

Finally, the total scores on the OPHI comparing adaptation in daily life before and after injury were significantly different $(p<0.005)$; lower adaptation scores were obtained post-injury. Indeed, statistically significant differences were found in each of the 5 areas covered in the interview (see Table IV), with the greatest changes observed in the scales measuring (1) identification and enactment of interests, values and goals, and (2) environmental influences. Closer examination of the results for the environment revealed that the major problem lay in the non-human environment; changes in family and social relationships (the human environment) were not statistically significant.

\section{Discussion and implications for therapy}

The results of the study indicate that the subjects' physical function improved over time, with some spontaneous recovery of ambulation and bowel and bladder function, as expected for persons with central cord injury.

The subjects' mean score on the ASIA Motor Index (81 out of a possible $100)$ suggests reasonably good overall motor function while the mean standard score on the JHFT indicates that the subjects had poor to very poor hand function. This demonstrates one of the main characteristics of the central cord syndrome. Because the ASIA Motor Index scale is a global measure of muscle 
action it may have limited usefulness in predicting function; the specific areas where loss of function has occurred cannot be inferred from the total ASIA score alone. For example it is conceivable that a person with central cord syndrome and a person with paraplegia would have the same ASIA score but their functional capabilities would be very different. Thus, for the ASIA Motor Index score to be clinically meaningful, it is necessary to view it in light of the type of lesion.

The results from the Klein-Bell ADL Scale reveal that the subjects function well in their activities of daily living. Although poor hand functioning would typically be predictive of difficulties in self-care and employment, subjects made effective use of adaptive equipment to compensate for limited hand function.

Fatigue was found to be an issue for most of the subjects, and may be a neglected side-effect of the syndrome. Thus, consideration should be given to incorporating energy conservation training into rehabilitation programmes for persons with central cord injuries.

Pain was also found to be a long term side-effect of central cord injury, yet most subjects did not take medication to relieve pain. Consequently, pain management techniques may also need to be considered as components of comprehensive rehabilitation programmes for the central cord injured population.

Finally, the results from the Occupational Performance History Interview demonstrate that the central cord injury significantly and negatively affected occupational performance in the following areas: organisation of daily living routines; maintenance of life roles; identification and enactment of interests, values and goals; and perception of ability and responsibility. For example a majority of the subjects experienced loss of the worker role. This loss seriously affected adaptation in the other spheres as exemplified by subject 8 who had been a logger before his injury. Since his injury his daily routine has changed from active participation to passive observation; he spends nearly all of the day watching television now. He feels unable to pursue the recreational interests he enjoyed prior to injury because they were mainly outdoor activities. Although he lives with his brother and sister-in-law he does not see himself as able to help around the house, nor does he feel any responsibility for doing so.

Subject 3 was retired before he sustained his injury thus he did not lose the major role of worker. Nevertheless his injury has prevented him from pursuing his interest in 'backyard farming' which involved taking care of pigs and chickens and mowing grass, etc. Because of his changed perception of his abilities he no longer participates in church activities though this was a strong interest before his injury.

Finally, since his injury, subject 10 has suffered a great deal of pain which interferes with his sense of self, his activities and his mood. At follow-up he was not able to think of anything he does well and he does not feel he can accomplish things he sets out to do. He is unemployed and does not see himself as ever returning to work. He does not see himself as able to help around the home and he sees himself as controlled by external influences. As the above suggest, he is quite depressed.

Pain and fatigue were significant factors in the daily lives of many of the subjects. Consequently, persons with central cord injuries may need assistance in learning how to reorganise and carry out their daily activities so as to achieve 
a balance in work, play, and functional routines; to maximise their participation in life roles; to re-estabish old interests or develop new ones; to appraise and re-establish values and goals; and to identify areas of responsibility in light of changes in ability related to the central cord injury. In addition, the results of this study indicate that more attention to modification of the physical environment may be needed.

Many of these issues become apparent only after the patient has been discharged home. Thus close follow-up care, including possible outpatient therapy for a period following inpatient discharge, should be considered.

\section{Acknowledgement}

The authors thank the American Occupational Therapy Association for permission to use the Occupational Performance History Interview-Research Version.

\section{References}

American Spinal INJURy Association 1982 Standards for neurological classification of spinal injury patients. ASIA, Chicago.

Bosch A, STAUfFer S, NiCKel V 1971 Incomplete traumatic quadriplegia: A ten year review. Fournal of the American Medical Association 19:216.

BURKe DC 1973 Pain in paraplegia. Paraplegia 10:297-313.

COHEN J 1960 A coefficient of agreement for nominal scales. Educational and Psychological Measurement 20:37-46.

Donovan WH, Dimitrijevic MR, DAHM L, et al. 1982 Neurophysiological approaches to chronic pain following spinal cord injury. Paraplegia 20:135-146.

GRUNDY D, RUSSELL J 1986 ABC of spinal cord injury: Later management and complicationsI. British Medical fournal 292:677-680.

HARDY AG, Rossier AB 1975 Spinal Cord Injuries, Orthopaedic and Neurological Aspects. Thieme, Stuttgart.

JeBSEN RH, TAYLOR N, TREischmann RB, et al. 1969 An objective and standardized test of hand function. Archives of Physical Medicine and Rehabilitation 50:311-319.

KIELHOFNER G, HENRY A 1988 Development and investigation of an occupational performance history interview. American fournal of Occupational Therapy 42(8):489-498.

KLEIN RM, BELL BJ 1982 Self-care skills: Behavioral measurement with Klein-Bell ADL Scale. Archives of Physical Medicine and Rehabilitation 63:335-338.

MERRIAM WF, TAYLOR TKF, RUFF SJ, et al. 1986 A reappraisal of acute traumatic central cord syndrome. The fournal of Bone and foint Surgery 68-B:5.

Nepomuceno C, Fine PR, RichardS JS, et al. 1979 Pain in patients with spinal cord injury. Archives of Physical Medicine and Rehabilitation 60:605-609.

PEARSON RG, ByARS GE 1956 The development and validation of a checklist for measuring subjective fatigue. School of Aviation Medicine, USAF, Randolph Air Force Base, TX.

SCHNEIDER RC, CHERRY G, PANTEK H 1954 The syndrome of acute central cervical spinal cord injury with special reference to the mechanisms involved in hyperextension injuries of cervical spine. Fournal of Neurosurgery 11:546-577.

TURK DC, RUDY TE 1987 Toward a comprehensive assessment of chronic pain patients: A multiaxial approach. Behavior Research and Therapy 25(4):237-249.

TURK DC, RUDY TE 1988 Toward an empirically-derived taxonomy of chronic pain patients: Integration of psychological assessment data. Fournal of Consulting and Clinical Psychology 56(2):233-238. 